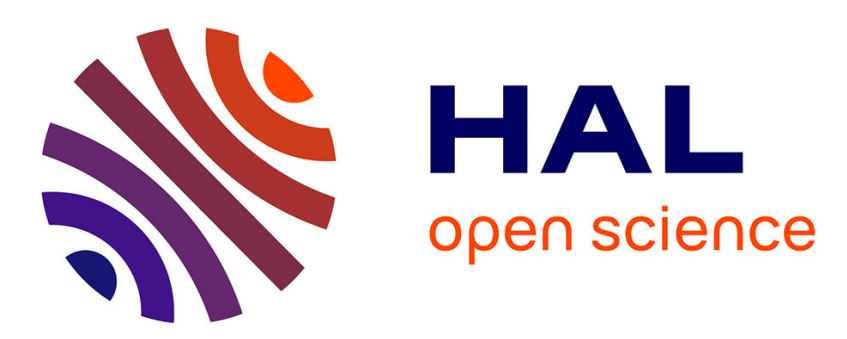

\title{
Analogical mapping across modalities in children with specific language impairment (SLI)
}

Sandrine Leroy, Christelle Maillart, Christophe Parisse

\section{To cite this version:}

Sandrine Leroy, Christelle Maillart, Christophe Parisse. Analogical mapping across modalities in children with specific language impairment (SLI). Research in Developmental Disabilities, 2014, 35

(9), pp.2158-2171. 10.1016/j.ridd.2014.05.005 . halshs-01009167

\section{HAL Id: halshs-01009167 \\ https://shs.hal.science/halshs-01009167}

Submitted on 27 Jun 2014

HAL is a multi-disciplinary open access archive for the deposit and dissemination of scientific research documents, whether they are published or not. The documents may come from teaching and research institutions in France or abroad, or from public or private research centers.
L'archive ouverte pluridisciplinaire HAL, est destinée au dépôt et à la diffusion de documents scientifiques de niveau recherche, publiés ou non, émanant des établissements d'enseignement et de recherche français ou étrangers, des laboratoires publics ou privés. 


\section{Analogical mapping across modalities in children with specific language}

\section{impairment (SLI)}

Sandrine Leroy ${ }^{\mathrm{a}}$, Christelle Maillart ${ }^{\mathrm{a}}$, Christophe Parisse ${ }^{\mathrm{b}}$

a Department of Psychology: Cognition and Behavior, University of Liege, B38, rue de 1'Aunaie, 30, 4000 Liège, Belgium

b Modyco-Inserm, University of Paris Ouest Nanterre la Défense, 200 avenue de la République, 92001 Nanterre, France

\section{Introduction}

The cognitive grammar approach to language acquisition proposes that grammar development is conceptualization (Langacker, 1987). Children develop their linguistic system by a gradual process of generalization from lexicalized forms (detected in linguistic input) to abstract forms (or construction schemas) (Bybee, 2010; Goldberg, 2006; Tomasello, 2003). According to Goldberg (1995, 2006) and Tomasello (2000, 2003), pattern-finding is the central cognitive construct in grammar acquisition. It allows children to go beyond linguistic input and create abstract linguistic schemas. This pattern-finding involves several general cognitive processes; one of them is analogical reasoning. Tomasello (2003) proposes that children make analogies across whole utterances to reach the more abstract dimension of linguistic competence. According to Gentner and Markman (1997), analogical reasoning makes it possible to generalize known forms to new linguistic contexts. Analogy is considered as a domain-general process which underlies the abstraction of linguistic forms and the construction of the linguistic system. The importance of analogy makes it likely that disordered analogical reasoning will have severe consequences on the abstraction of 
construction schemas, entailing poor productivity and creativity with language and a greater dependency on linguistic input.

Children with specific language impairment (SLI) present a linguistic developmental pathology characterized by the slow development of spoken language. Their language difficulties cannot be explained by hearing loss, late motor development or global cognitive disorders (Leonard, 1998; Schwartz, 2009). Furthermore, children with SLI do not present with other neurodevelopmental disorders such as autism nor do they suffer from intellectual and emotional impairments (Leonard, 1998; Schwartz, 2009). Although children with SLI present varied language profiles, a common profile in children with SLI is a mild to severe deficit in at least two language areas and a generally greater weakness in the morphosyntactic component (Schwartz, 2009). Several authors have observed that the difficulties of children with SLI are characterized by a lack of syntactic productivity and a greater input dependency. These observations are in agreement with the hypothesis of an impaired ability to generalize language forms. As argued by Riches, Faragher and Conti-Ramsden (Riches, Faragher, \& Conti-Ramsden, 2006): “(...) these children resemble children at a younger language stage in terms of their syntactic creativity and their tendency to use rote-learned forms". We speculate that this lack of generalization may be underlain by a deficit in analogical reasoning, and more particularly by a deficit in analogical mapping.

In this paper, we hypothesize that analogical reasoning, and more particularly the process of mapping, is problematic in children with SLI. We inquire whether children with SLI are able to detect a relational similarity between several elements within sequences and to transfer this learning to a novel sequence of elements. Further, we surmise that children with SLI will have greater difficulty detecting relational similarities in verbal sequences (which involve linguistic processing) than in non-verbal sequences (which involve visual processing). If children with SLI have difficulties finding the similar relational structure between two 
situations and using it with other elements, this could explain their lack of syntactic creativity and it could thus explain the lack of generalization of construction schemas and a greater dependency on linguistic input.

\section{Analogical reasoning in children with SLI}

Until now, most of the studies that have addressed analogical reasoning in children with SLI sought to explore the influence of cognitive and linguistic abilities on analogical reasoning tasks (Masterson, Evans, \& Aloia, 1993; Nippold, Erskine, \& Freed, 1988). Analogical reasoning was assessed by using the "A:B::C:D" paradigm. Children had to deduce the relation between the first two items $(\mathrm{A}: \mathrm{B})$ and then transfer this relation to another pair of items (C:D). Used items were utterances or words as well as geometric shapes. Nippold et al. (1988) investigated analogical mapping in 20 children with SLI, aged 6-8 years, compared to age-matched children with typical language development (TLD). In the first task, children heard utterances and had to compare them in order to produce a new fourth form (e.g. Warm (A) goes with cold (B) just as happy (C) goes with...? (D) - Response: unhappy). In the second task, the instructions were the same but the items used were geometric shapes. Results indicated that children with SLI performed more poorly than children with TLD on both tasks. However the group effect disappeared when the data were reanalysed by controlling for nonverbal intelligence score, suggesting that nonverbal abilities underlie these analogical reasoning tasks. Masterson et al. (1993) investigated the influence of cognitive and linguistic abilities on the performance of children with SLI using five types of verbal 
analogical reasoning tasks: synonyms (e.g. easy:simple::shut:?); antonyms (e.g. cool:warm::black:?); linear order (e.g. puppy:dog::cub:?); category membership (e.g. red:colour::hit:?); and functional relationship (e.g. horn:play::horse:?). The performance of children with SLI was compared to the performance of two groups of normally developing children: a group of mental-age matched peers - matched on nonverbal intelligence - and a group of language-age matched peers - matched on their lexical production score. Children with SLI performed more poorly than their mental age-matched peers on all of these tasks. Moreover, although children with SLI had a higher mental age than their language-matched peers, this difference did not help children with SLI, who always performed less well than their language-matched peers (except for antonyms). Consequently, when nonverbal abilities are controlled, the results obtained in the two studies are contradictory.

As mentioned by Masterson and Perrey $(1999, p$. 5) "These studies indicate that knowledge of vocabulary, sufficient exposure to the type of relationship used in an analogy and the ability to see the similarity between analogy components are three potential problems for the solution of analogies by children with language disorders". In these tasks, the resolution of problems requires not only more general cognitive processes (such as working memory or inhibitory control), but also linguistic knowledge. The semantic component plays a fundamental role because different kinds of semantically-based implicational relationships are involved. Moreover, these tasks required considerable metalinguistic thought. Consequently, the performance of children with SLI in these verbal reasoning tasks may not specifically reflect a problem with the processes required in analogical reasoning, but could be the consequence of poor linguistic knowledge. In this paper, we study the influence of analogical mapping, which is the core process of analogical reasoning, on the language development of children with SLI. Analogical mapping plays a fundamental role in language development. Although the literatures on schemas and analogy were previously largely 
independent, recent theoretical considerations have envisaged the implication of analogical mapping in the abstraction of construction schemas.

\section{Analogical mapping, the core of analogical reasoning}

Reasoning by analogy requires three processes (Gentner \& Colhoun, 2010; Gentner \& Smith, 2012). These processes are (1) retrieval that is the process allowing the retrieval from long term memory of a prior situation analogous to a current situation present in working memory; (2) mapping that involves a process of aligning the representations of the two analogous situations in working memory and projecting inferences from one situation to the other; and (3) evaluation that involves a judgement of analogy and its inferences when analogical mapping has been accomplished. Gentner and Smith (2012) stress that mapping is the core process in analogical reasoning. While analogical reasoning does not systematically involve finding a second similar situation in memory, it invariably involves a mapping process.

Gentner (1983) considers that analogical mapping requires two steps that are structural alignment and projection of inferences. During the first step, two analogous situations are aligned on the basis of their common relational structure. This is called structural alignment. According to Gentner and Smith $(2012, p$. 130), "reasoning by analogy involves identifying a common relational system between two situations and generating further inferences driven by these commonalities". Consequently, an overlap in the relational structure of the two situations is essential whereas concrete property matches between the two situations being analogized is not necessary. When relations between objects are compared through situations, the properties specific to the objects are ignored whereas relations between objects are preserved. The second step in analogical mapping consists in projecting inferences from the 
previous situation to the new situation. If a part of a new situation is not present, the learner is able to choose a candidate inference for completing this missing pattern when the two analogous situations are aligned and when their common relational structure is found.

Analogical mapping is the result of a developmental trajectory characterized by two different strategies for 6- and for 14-year-olds (Richland, Morrison, \& Holyoak, 2006; Thibaut, French, \& Vezneva, 2010). Younger children use the first strategy, giving priority to object matches (i.e. common perceptual elements). They choose the first stimulus that is perceptually the most salient solution (Richland et al., 2006; Thibaut et al., 2010). Older children use the second strategy and give priority to relational similarity, and thus to analogical mapping. Between these two steps, there might be "real conflicting situations in which children (...) consider various possibilities, including perceptual and relational matches" (Thibaut et al., 2010: 3). The shift from a focus on object matches form a focus on relational matches is called relational shift (Rattermann \& Gentner, 1998). Young children align exemplars which share perceptual similarities because they common matches are automatically aligned. Consequently, while perceptual similarity is not necessary for reasoning by analogy, it helps for abstracting construction schemas. Alignment of exemplars that share perceptual similarity helps in discovering relational similarity between exemplars that are more distant. During this progressive alignment (Gentner \& Colhoun, 2010) concrete and dimensionally specific representations become more abstract by comparison and alignment.

Three hypotheses could explain age-related differences in the selection of the strategy used to compare two analogous situations. The first hypothesis is that, given that analogical mapping is correlated with the knowledge about relevant relations, the greater the knowledge about relevant relations, the more efficient the analogical reasoning will be (Rattermann \& Gentner, 1998). The second hypothesis is that the shift results from a developmental increase 
in processing capacity. In this case, processing relational matches requires more processing than processing object matches and younger children are thought not to have sufficient processing capacity for processing relational matches (Halford \& Andrews, 2011). The third hypothesis considers the development with age of working memory capacity (Cho, Holyoak, \& Cannon, 2007; Morrison, Holyoak, \& Truong, 2001) and inhibitory control (Morrison, Doumas, \& Richland, 2006; Richland, Chan, Morrison, \& Au, 2010; Richland et al., 2006; Thibaut et al., 2010). Working memory is inseparable from analogical mapping bevause carrying out analogical mapping requires learners to maintain and manipulate information in order to infer relational similarities between two situations (Gentner, 1983; cited by Richland et al., 2006). Moreover, inhibitory control is required when relational and perceptual responses come into conflict (Richland et al., 2010; Richland et al., 2006; Viskontas, Morrison, Holyoak, Hummel, \& Knowlton, 2004). Children have to resist responding on the basis of superficial features and have to focus on responding on the basis of relational correspondences between the structures analysed.

\section{Analogical mapping and pattern-based abstraction}

Recent theoretical considerations have envisaged the implication of analogical reasoning in language development. For example, research in child language development from a usagebased perspective shows that the abstraction of construction schemas and categories is possible if children are able to detect relational patterns between two or several utterances (Tomasello, 2003). Studies into pattern-based abstraction suggest that analogical mapping and early language development may be linked. Indeed, analogical mapping is required in early language development by allowing pattern-based abstraction. Pattern-based abstraction can be described in terms of "relational operations over physical stimuli in sequence" (Gómez \& 
Gerken, 2000). This pattern-based abstraction is used by children to acquire structured sequence regularities, including word order regularities in addition to words. Children use these regularities to develop their language.

Pattern-based abstraction can be investigated by means of rule-learning tasks that study infants' linguistic rule learning. Marcus et al. (1999) inquired into infants' ability to generalize their knowledge about the rules governing syllable combinations. Infants aged seven months were presented with triads of syllables that were organized in ABA sequences (e.g., [ga-ti-ga]) or ABB sequences (e.g., [ga-ti-ti]). Half of the infants were familiarized with ABA sequences while the other half were familiarized with $\mathrm{ABB}$ sequences. During the test phase, the infants were tested with new triads of syllables that shared either the familiar pattern or the novel pattern. The infants were able to discriminate successfully between familiar patterns and novel patterns when these were presented using new syllables. Consequently, infants readily acquire these rules when they are presented with linguistic stimuli. Although Marcus et al. (1999) did not obtain the same results with non-linguistic stimuli, Saffran, Pollak, Seibel and Shkolnik (2007) showed that 7-month-old infants can detect and generalize these same patterns when the elements consist of pictures of animals (cats and dogs). These findings indicate that rule learning of this type is not specific to language acquisition and, consequently, not domain-specific. These rule-learning tasks are more than just simple sequence learning because they involve generalization. Infants must do more than learn the token-level patterns (Saffran et al. 2007). The learner must detect the same/difference relationships within sequences, requiring the learner to represent and categorize sequence tokens as being of the same or different type. If they stopped at the tokenlevel patterns (e.g. "Malamute" - "Cattle Dog" - "Malamute", see Saffran 2007) and did not discover the abstract pattern ABA, they would be unable to perform the task correctly. Analogical mapping underlies pattern-based abstraction. Children have to detect a relational 
structure between elements of the sequences in order to abstract a relational pattern that will make generalization to new items possible.

\section{Analogical mapping in children with SLI}

Analogical mapping is required in language development. Consequently, a disordered analogical mapping could explain why children with SLI are unable to transfer their linguistic knowledge to new situations that are structurally and relationally similar to already known situations. Children with SLI, it is suggested have more difficulty generalizing linguistic forms, show a lack of variability and productivity in language use, and are more input dependent (e.g. Jones \& Conti-Ramsden, 1997; Riches et al., 2006; Skipp, Windfuhr, \& Conti-Ramsden, 2002; Thordardottir \& Weismer, 2002).

Until now, only one study has attempted to establish a link between analogical mapping and language disorders in children with SLI (Leroy, Parisse, \& Maillart, 2012). The authors investigated the ability of children with SLI to discover relational structure between two sequences and to infer this structure in another sequence. This task involved geometric shapes so as to limit the interference of linguistic knowledge. After being presented with two sequences that shared the same relational matches with more or less perceptual similarities, children had to complete a third sequence that was relationally similar to the first two sequences. Results showed that children with SLI had poorer results than children with TLD (typical language development), indicating that children with SLI have greater difficulty detecting the relational similarity between sequences composed of shapes when the perceptual similarity between elements in the sequences decreases. These problems with analogical mapping could reflect the difficulty of children with SLI with pattern-based abstraction and their difficulties in generalizing a common relational pattern to new items. 


\section{Aim of the study}

In their study, Leroy et al. (2012) used only non-linguistic sequences to investigate analogical mapping. The current study compares children's performance between two tasks: a non-linguistic task and a linguistic task. Analogical mapping is a domain-general process that is required in all the situations involving an overlap in relational structure between two situations in order to generate further inferences. Consequently, analogical mapping is required in non-linguistic modality (e.g. to solve a mathematical problem) as well as in linguistic modality (e.g. to abstract construction schemas). Given their language disorders, we predict that children with SLI will have more difficulties processing linguistic information compared to non-linguistic information.

Moreover, Leroy et al. (2012) used geometric shapes that might require semantic knowledge. Consequently, it could be suggested that children with SLI did not benefit from the language-based knowledge and reasoning that may have been used by children with TLD to solve the task. In the current study, pictures with little semantic content were used in the non-linguistic task. The goal was to investigate analogical mapping without the involvement of linguistic knowledge. In the linguistic task, syllables were used because they do not require lexical knowledge and they do not activate preliminary linguistic knowledge. Creating a nonlinguistic task that shares the same characteristics as the linguistic task (i.e. sequential presentation and no lexical knowledge) allows better identification of disordered processes in children with SLI. If the difficulties in children with SLI are a consequence of auditory disorders, an effect of modality (linguistic vs. non-linguistic) is expected, with better performance for the non-linguistic task. On the contrary, if children with SLI have difficulties 
with the alignment of elements, which involves extracting regularities from sequential material, their difficulties can be expected to occur in both modalities.

In the current study, we were also interested in the strategies used by children with SLI to detect relational similarities between two sequences and to infer this structure in a third sequence. According to some authors (Richland et al., 2006; Thibaut et al., 2010), discovering relational similarity between several constructions is the result of a developmental trajectory characterized by two different strategies. Whereas younger children preferentially use perceptual similarities, older children mainly use relational matches. Our prediction is that children with SLI, as is typical of young children, will preferentially use a perceptual strategy and that they will be more dependent on perceptual similarity to detect relational similarity between sequences than their age-matched peers.

\section{Methodology}

Participants

Nineteen monolingual French-speaking children with SLI (4 girls and 15 boys; aged $8 ; 10$ to $11 ; 9)$ participated in the study. This ratio male/female is representative of clinical sample. Several studies have reported higher rates of SLI among males than among females, with an estimating ratio from 2:1 to 3:1 (Bishop, 1997; cited by Whitehouse, 2010). Children with SLI were recruited in "language classes" attached to specialized primary schools, in the French-speaking part of Belgium. Language class is defined as a specialized class for children with SLI where the educational focus is on the development of language skills. Informed consent was obtained from the parents of all participating children. A medical history questionnaire was completed by the parents. Questionnaire allows us to verify that all children were French native speakers, suffered no neurodevelopmental delay or sensory impairment, 
and had no history of psychiatric or neurological disorders. Parents confirm us that their child had normal hearing, vision, oral and speech motor abilities. Prior to the study, children with SLI were diagnosed as presenting with SLI by speech-language pathologists and child neurologists. Moreover, we used standard clinical tests to ensure that all of the children with SLI met criteria generally adopted in scientific literature. Children with SLI scored more than -1.25 SD below expected normative performance in at least 2 language components (according to the SLI criteria adopted by Leonard et al., 2007), most notably a deficit in productive grammatical abilities. The children's language abilities were assessed with several tests frequently used by French speech-language therapists: the Echelle de Vocabulaire en Images Peabody (EVIP; Dunn, Theriault-Whalen, \& Dunn, 1993- a French adaptation of the PPVT-R), two subtests of the Evaluation du Langage Oral (ELO: French Language Evaluation - Khomsi, 2001 - the word repetition and the utterance production subtests), and the Epreuve de Compréhension Syntaxico-Sémantique (ECOSSE; Lecocq, 1996). Furthermore, all of the children with SLI had a non-verbal intellectual quotient of 82 or greater (nonverbal IQ was measured by using the Perceptual Reasoning Index (PRI) of the WISC IV (Wechsler, 2005).

Nineteen monolingual French-speaking children with typical language development (TLD) (5 girls and 14 boys; aged from 8;10 to 11;9 years) were also recruited for the current study. They came from France. Their linguistic performances were controlled and all of them were at or above age-level expectations. Children with TLD were matched on children with SLI on the basis of the perceptual reasoning index ( $+/-8$ points) and on the chronological age (+/- 5 months). The two groups differed neither on the perceptual reasoning index nor on chronological age. However, both groups were significantly different on all standardized measures of language (see Table 1).

- Insert Table 1 about here - 
Design

Analogical mapping was investigated by means of two pattern-based abstraction tasks: a linguistic task and a non-linguistic task. We deliberately chose pattern-based abstraction that does not require linguistic knowledge or knowledge of form-meaning pairings in order to test the basic processes involved in analogical mapping (i.e. to infer a relational structure without taking meaning into account and generalize this structure to new items). Children had to focus only on formal aspects. Thus, this basic process of pattern-based abstraction has repercussions on language development.

The two tasks were composed of 36 items. An item was composed of three three-unit sequences: two reference sequences (considered as priming sequences) and one test sequence (considered as the target sequence). In these tasks, children had to choose two elements (pictures in the non-linguistic task; or syllables in the linguistic task) among four different elements in order to complete a third sequence (test sequence). The children had to complete the test sequence according to the particular logical relation between the units of the sequences being analogized. In the two tasks, two types of items were created: items with perceptual similarity and items with no perceptual similarity. Items with perceptual similarity were characterized by the presence of object matches, which are perceptual factors, between the sequences being analogized. In contrast, in items without perceptual similarity, there were no object matches between the three sequences, so children had to rely on relational similarity.

\section{Linguistic task}

\section{Items}


The three sequences were composed of three auditory CV syllables. Eleven consonants and six vowels were selected. The chosen syllables were very distinct and perceptually discriminable in order to avoid wrong answers being due to difficulties in phonological discrimination (difficulties reported in children with SLI; Elliott, Hammer, \& Scholl, 1989; Stark \& Hienz, 1996; Sussman, 1993). To select highly discriminable syllables, we used Costerman's table (1980, p. 86) in which the author analyses the average subjective evaluations of 70 participants for consonant pairs. Participants had to listen to each pair and repeat it (e.g. $[p]-[t]$ ). Participants had to evaluate for each pair the degree of difference between the two consonants by a number from 1 (weak difference) to 6 (strong difference). We chose only those pairs considered as the most discriminable (e.g. [p] vs. [n]) in Costerman's table.

\section{Description of the task}

During the two reference sequences, syllables were presented one after another every 500 milliseconds. Visual cues were presented on the screen to indicate the place of each syllable in the sequence and the end of the reference sequences. Children had to complete the test sequence by discovering the similarity that linked the two reference sequences. Reference sequences were presented twice before the test sequence appeared. This presentation of several exemplars facilitated discovery of the relational match that linked the elements (Tomasello, 2003). Repetition of the information also allowed a reduction in the cognitive overload in memory (see Figure 1).

- Insert Figure 1 about here - 
Children were asked to create a test sequence that shared relational similarities with the two reference sequences. Only the first syllable of the test sequence was presented to the children. To complete the test sequence, children were asked to choose which two syllables, among four possible responses ([lo], [li], [mo], [mi]), 'went best'. Each of the possible responses was linked to a specific keyboard key. In order to avoid the child pressing an undesired key, a typing mask was placed on the keyboard. On this typing mask, written syllables corresponding to the four possible solutions were placed next to the associated key. Before beginning the task, children's reading of the four syllables was controlled.

In order to investigate the influence of perceptual similarity on children's performance, perceptual cues between sequences decreased during the task. The perceptual cues used between sequences in the linguistic task were consonants (consonants were the same in the three sequences; e.g. first reference sequence [ma-la-ma] - second reference sequence $[m u-l u-m u]$ - test sequence $[m i-?(l i)$ - ?(mi)]) or vowels (vowels were the same in the three sequences; e.g., [bi-bo-bi] - [si-so-si] - [mi-?(mo)-?(mi)]). The first items of each type of sequence shared perceptual similarity and relational similarity whereas the last items essentially shared relational similarity only between sequences of pictures (see Figure 2). This order of items allows progressive alignment that facilitates detection of relational patterns.

- Insert Figure 2 about here -

\section{Non-linguistic task}

\section{Items}

The design of the non-linguistic task was the same as the linguistic task, with the difference that sequences were composed of three pictures that had little semantic content (cf. 
Appendix 1). These pictures came from the study by Kroll and Potter (1984). In order to select the pictures that had the least semantic content, a questionnaire with 35 pictures was distributed to 70 adults. By means of a Lickert scale from 1 (Strongly disagree) to 5 (Strongly agree), adults had to determine if the pictures looked like a real object. After analysis, we kept 11 pictures for which all the adults had responded "Strongly disagree" or "Disagree".

These pictures were presented in six colours (red, blue, green, yellow, black, purple). Thus, two features were considered: shape and colour. Size was not used because this dimension is less salient and thus more cognitively demanding (Marshall, 2003). In the same sequence, the chosen pictures were discriminable in both shape and colour, in order to avoid any confusion between them. To compare the results of the verbal and non-verbal tasks, we took care to propose the same distribution of relational and perceptual similarities between the two tasks. A consonant in the verbal task was always associated to a specific form (e.g., the consonant $[b]$ was associated to the form specific colour (e.g., the vowel [a] was associated to the colour blue).

\section{Description of the task}

The design of the task was similar to the linguistic task. During the two reference sequences, pictures appeared on the screen one after another every 500 milliseconds. Visual cues were presented on the screen to indicate the end of the reference sequences. Children had to complete the test sequence by discovering the similarity that linked the two reference sequences (see Figure 1). Reference sequences were presented twice before the test sequence appeared. As in the linguistic task, children were asked to complete the test sequence by choosing the two pictures, among four possible responses, that 'went best', in comparison with the two reference sequences (see Figure 3). Each of the possible responses was linked to 
a specific keyboard key. A typing mask was placed on the keyboard on which pictures corresponding to the four possible solutions were placed next to the associated key.

\section{- Insert Figure 3 about here -}

The number of items and their order of presentation was the same as in the verbal task. The complexity of the task was progressive. The complexity was associated to the degree of object commonalities between the three sequences. In this non-linguistic task, two dimensions were considered for perceptual cues: colour and shape (see Figure 4).

- Insert Figure 4 about here -

\section{Procedure}

Participants were tested individually in an isolated room. For the linguistic task, headphones were used. Before the experimental tasks, children were administrated two tasks of perceptual discrimination. The first task was a visual discrimination task that consisted in the sequential presentation of 30 pairs of pictures. These pictures were the same as those used in the non-linguistic task. The second task was an auditory discrimination task in which 30 pairs of syllables were presented to the children. The syllables were the same as those used in the linguistic task. For the two discrimination tasks, children had to verbally judge if the two stimuli presented in a pair were the same or not. The threshold of correct responses was fixed at $80 \%$ (24 correct responses out of the 30 pairs). If a child did not reach the threshold, the experimental tasks were not proposed. The goal of these discrimination tasks was to ensure 
that any difficulties of the children with SLI in the experimental tasks did not result from problems discriminating stimuli.

The two experimental tasks were administered to the children during 2 sessions. EPrime software (Schneider, Eschman, \& Zuccolotto, 2002) was the computer programme used to run the experiment. Prior to the testing phase, children were submitted to training trials that allowed them to become familiar with the task and the instructions. Following the practice trials, children were presented with the testing task. The tasks lasted about 1 hour. The task was inserted inside a story and rewards were awarded every six items in order to maintain the children's attention during the whole testing phase.

\section{Results}

No child was excluded from the analyses as all children responded above chance level. Moreover, all the children understood the instructions and were able to successfully complete the practice trials. A point was given when the response was correct.

A repeated-measures analysis of variance 2 (Modality: Linguistic vs. Non-linguistic) X 2 (Perceptual support: with perceptual cues vs. without perceptual cues) with the group (SLI vs. TLD) as independent variable was performed. Results indicated a significant main effect of $\operatorname{Group}\left(F(1,36)=18.98, p<0.001\right.$, partial $\left.\eta^{2}=0.35\right)$, with overall performances for the children with SLI $(M=12.84 ; S D=4.06)$ being poorer than that of their age-matched peers $(M=16.47 ; S D=1.64)$. Statistical analyses revealed a significant main effect of Modality $\left(F(1,36)=13.15, p<0.001\right.$, partial $\left.\eta^{2}=0.27\right)$, with poorer performance for the 
linguistic modality $(M=13.95 ; S D=3.72)$ than for the non-linguistic modality $(M=15.36$; $S D=3.33)$. Results indicated no significant main effect of Perceptual support $(F(1,36)=$ $0.37, p>0.05$, partial $\eta^{2}=0.01$ ). Children's performances were the same for the items with $(M=14.75 ; S D=3.37)$ or without $(M=14.57 ; S D=3.81)$ perceptual cues.

Interestingly, no significant Group X Modality interaction was revealed $(F(1,36)=$ $0.45, p>.05$, partial $\left.\eta^{2}=0.012\right)$.. Moreover, results indicated no significant Group X Perceptual support interaction $\left(F(1,36)=2.2, p>.05\right.$, partial $\left.\eta^{2}=0.06\right)$. Although children with SLI performed more poorly than children with TLD, the differences between the two groups were not more marked for items without perceptual cues. Interestingly, a significant Group X Modality X Perceptual support interaction $\left(F(1,36)=5.43, p<0.05\right.$, partial $\eta^{2}=$ 0.13) (See Figure 6) was revealed. Whereas, in non-linguistic modality, children with SLI had the same performance for items with and without perceptual cues $\left(F(1,36)=0.44, p>0.05, \eta^{2}\right.$ $=0.01$ ), they performed more poorly with items without perceptual cues than with items with perceptual cues in linguistic modality $\left(F(1,36)=8.71, p<0.01, \eta^{2}=0.19\right)$. In contrast, children with TLD had the same performance with items with and without perceptual cues, both in the non-linguistic modality $\left(F(1,36)=0.44, p>0.05, \eta^{2}=0.01\right)$ and in the linguistic modality $\left(F(1,36)=0.15, p>0.05, \eta^{2}=0.004\right)$.

- Insert Figure 5 about here -

\section{Discussion}

In this study, we aimed to investigate the ability of children with SLI to generalize a relational pattern to new items. A rule learning task was used in order to avoid interference of language knowledge that could hinder the performance of children with SLI compared to children with TLD. This task allows pattern-based abstraction to be studied in children with 
SLI. In other words, it assesses children's abilities to detect a common relational pattern between sequences and to generalize it to new items.

\section{Impact of auditory processing}

Two modalities (linguistic and visual) were proposed to investigate the impact of auditory processing on the performance of children with SLI to this type of task. Several authors (e.g. Benasich \& Tallal, 2002; Tallal, 1990) consider that the language disorders of children with SLI can be explained by a deficit in auditory processing. They argue that children with SLI have specific difficulties during the processing of rapid sequential information. If this is the case, we could expect poorer performance of children with SLI in the linguistic task compared to the non-linguistic task.

Results showed that all the children, children with SLI as well as children with TLD, performed better for the non-linguistic task than for the linguistic task. Working memory is strongly required in this task (children had to maintain information in memory before generalizing the structural pattern to new items). Cohen and colleagues (Cohen, Evans, Horowitz, \& Wolfe, 2011; Cohen, Horowitz, \& Wolfe, 2009) have demonstrated that auditory memory is systematically poorer than visual memory in adults. They put forward two possible explanations for this difference between the two modalities. According to the first hypothesis, the physics or psychophysics of auditory objects may be fundamentally different from those of visual objects. Auditory objects might be less memorable than their visual counterparts. According to the second hypothesis, auditory memory might be fundamentally different, i.e. smaller, than visual memory. A better performance by children in a non-linguistic task compared to a linguistic task has already been reported in a study by Gabriel et al. (2013). By studying procedural learning across visual and verbal modalities, the authors showed that all 
the children (with and without SLI) in the study processed visual stimuli more quickly than auditory stimuli.

\section{Impact of combination between auditory processing and perceptual support}

We expected two interaction effects that were not confirmed by the statistical analyses. No significant interaction effect between group and modality was revealed, showing that auditory processing did not increase the difficulties of children with SLI. Moreover, no significant interaction effect between group and perceptual support was found, showing that children with SLI did not seem to benefit from the presence of perceptual similarity.

In fact, a combination of auditory modality and absence of perceptual similarity appeared to cause more difficulties in children with SLI, since a very interesting triple interaction Group*Modality*Perceptual Support effect was found. Children with SLI had the same performance in the non-linguistic task for items with and without perceptual cues but they performed less well than children with TLD for items without perceptual cues compared to items with perceptual cues in the linguistic task. Children with SLI have difficulties with visuo-spatial memory but these are less marked than their difficulties with verbal memory (for a review, Vugs, Cuperus, Hendriks, \& Verhoeven, 2013). The retention of verbal information is consequently more disordered. This difference between verbal and visual information does not appear with items with perceptual similarity but only with items without perceptual similarity. Consequently, the absence of perceptual similarity in linguistic items seems to involve an additional cognitive load that negatively impacts on the performance of children with SLI.

"The general prediction of structural alignment is that similarity comparisons lead subjects to attend to the matching relational structure in a pair of items" (Markman \& 
Gentner, 1993, p. 431). This is called progressive alignment (Gentner \& Colhoun, 2010). In agreement with this postulate, we manipulated perceptual similarity by proposing sequences with perceptual similarity before sequences without perceptual similarity. We decided to adopt a progressive complexity from more to less perceptual cues available. This design aims to facilitate the detection of relational structure between sequences and, consequently, to help children in performing this task. The first items help children to compare sequences more easily. Little by little, the children learned to compare sequences even if perceptual cues decreased. According to our results, children with SLI appear to benefit from this progressive alignment with visual items. However, when perceptual similarity decreases, verbal items are more difficult to process. The impact of progressive alignment with linguistic items seems less effective.

These results may reflect a cognitive overload linked to the combination of auditory processing and absence of perceptual similarity. In fact, analogical mapping is strongly influenced by processing load (Gentner \& Smith, 2012) and performances in children with SLI seem to be especially affected by processing load (e.g. Finneran, Francis, \& Leonard, 2009; Montgomery \& Evans, 2009; Oram Cardy, Tannock, Johnson, \& Johnson, 2010). More cognitive resources are used to maintain verbal information in memory (compared to visual information) and detect relational structure without perceptual cues (compared to the presence of perceptual cues) and, consequently, fewer resources are available to transfer this structure to new items. Developmental increase in processing capacity helps analogical mapping (Loewenstein \& Gentner, 2001). The results obtained here are compatible with this postulate. However, even if it is attractive, this hypothesis does not explain all the results. For it to be confirmed, the performance of children with SLI would have to be better with visual items with perceptual similarity because these items require less cognitive load than other conditions. 


\section{Role of processing capacities}

Overall, the performance of children with SLI is weaker than that of children with TLD in this task. Consequently, children with SLI have difficulties with analogical mapping compared to children with TLD. These results corroborate the results of the study by Leroy, Parisse and Maillart (Leroy et al., 2012). To understand this difference between the two groups, working memory capacity (Cho et al., 2007; Morrison et al., 2001) and inhibitory control (Morrison et al., 2006; Richland et al., 2010; Richland et al., 2006; Thibaut et al., 2010) were considered.

Children with SLI have difficulties with working memory (Isaki, Spaulding, \& Plante, 2008; Marton, Kelmenson, \& Pinkhasova, 2007) that is inseparable from analogical mapping (Cho et al., 2007; Morrison et al., 2001). Children had to maintain elements in memory and had to manipulate them in order to complete the third sequence. Consequently, the role of working memory on children's performance in these tasks is considered. Children with SLI had a poorer reverse span than their peers with TLD. However, within the two groups, there was no correlation between performance and reverse span. In other words, in each group, children with poorer reverse span did not necessarily perform more poorly in either of the two modalities (linguistic vs. non-linguistic). Thus, the poorer performance of children with SLI cannot be completely explained by a lower span of children with SLI.

Children with SLI have difficulties with inhibitory control (Bishop \& Norbury, 2005; Im-Bolter, Johnson, \& Pascual-Leone, 2006). Friedman and Miyake (2004) envisaged three functions of inhibition. The first function consists in the suppression of an automatic response. To avoid dominance of a structural pattern and, consequently, an automatic 
response, the three different structural patterns (i.e. ABA-ABB-BBA) were randomly and equitably distributed. The second function consists in the resistance to proactive interference (to inhibit more pertinent information). There is no reason to believe that earlier learning could have an impact on performance in these tasks and children had no reason to believe that one response was more pertinent than another because no feedback was given. The third function consists in the resistance to interference of distractors (i.e. to inhibit non pertinent information). In these tasks, no distracting information was inserted. Children had to take account of all the features of all the presented syllables and pictures to complete the testsequence correctly. However, some inhibitory difficulties due to the visual processing of very complex stimuli cannot be ruled out. Moreover, to infer the relational structure that links the different elements in the sequences, children have to inhibit perceptual features which are more salient information. This may indicate that children with SLI are unable to generalize a relational structure to new items because of their difficulties in inhibiting more salient possibilities that are unavailable among the possible solutions. Even if the role of inhibitory control in analogical mapping tasks in children with SLI has not yet been demonstrated, the role of inhibition cannot be neglected and could influence the performance of children with SLI.

\section{What about relational knowledge?}

In their study, Goldwater, Tomlinson, Echols and Love (2010) suggest that the inability of children aged 4 years to map syntactic relations is not due to processing capacity limits but rather to a dearth of relational knowledge (Rattermann \& Gentner, 1998). This point of view suggests envisaging relational knowledge in children with SLI and its influence on 
their performance. Relational knowledge can be defined as knowledge of the relation that links objects with one another.

Johnston and Smith (1989) showed that children with SLI, like children with TLD, performed at near ceiling level when they had to select the same picture as that chosen by two experimenters. Moreover, they had no difficulty in selecting the two identical pictures from a set of three. However, children with SLI were impaired on a more abstract task involving dimensional matches. Children had to detect which of two dimensions, colour or size, linked the two items chosen by the experimenters. The children's performances were poorer when the chosen pictures were linked by size but performance was improved when colour was considered. The effective use of the available dimension by children with SLI was impeded. We can deduce that detecting relations between elements is difficult for children with SLI when it involves dimensional matches. This may explain why children with SLI perform less well than children with TLD, especially for the non-linguistic task in which dimensional matches (i.e. color and shape) were considered for detecting relational structure between sequences.

\section{Limits of the study}

One limit of this study concerns response modality. As a reminder, to complete the test-sequence, children could choose four possible elements. Each of the possible elements was linked to a specific keyboard key. In order to avoid the child pressing an undesired key, a typing mask was placed on the keyboard. On this typing mask, perceptual cues (picture for the non-linguistic task and written syllables for the linguistic task) corresponding to the four possible solutions were placed next to the associated key. We decided to adopt this procedure to allow comparison between the results of the two tasks. We had to choose the same response 
modality for the two tasks. However, some children used an unexpected strategy to complete the test-sequences. For items with perceptual similarity, children pressed the two keys that shared perceptual similarity. This strategy reduced cognitive load. For linguistic items, these visual cues can help children and improve their performance for items with perceptual similarity that could explain the absence of an interaction effect between children with SLI and children with TLD.

\section{Future perspectives}

This study falls within the scope of usage-based theories according to which language development depends on a gradual mechanism of generalization from concrete lexicalized forms to more abstract construction schemas. Analogical mapping underlies this mechanism of generalization and, consequently, language development (cf. Gentner \& Colhoun, 2010; Gentner \& Namy, 2006). Children with SLI have language difficulties that seem linked to a lack of ability to generalize. Consequently, studying the integrity of analogical mapping in children with SLI is an interesting area of future research in language pathology.

Processing capacities and relational knowledge have been proposed to explain the poorer performance of children with SLI compared to children with TLD, and more particularly for linguistic items that did not share perceptual similarity. Indeed, it would seem that auditory modality linked to the absence of perceptual similarity may cause a cognitive overload to which children with SLI are particularly sensitive because of their limited processing capacities. Working memory and inhibition seem to be two interesting directions for investigation. More research is necessary to understand their impact on the performance with children with SLI better (for example, by proposing task with perceptual distractors - cf. Thibaut et al., 2010). 
The absence of perceptual effect suggests that a presentation of items with perceptual similarity could help to detect relational structure, thanks to progressive alignment. A task in which a random presentation of items is compared to a progressive progression could clarify the role of progressive alignment in children with SLI.

One important variable was not considered here: the frequency of exemplars. The mechanism of generalization, and consequently the abstraction of abstract schemas based on relational structure, requires the alignment of a certain number of exemplars (Tomasello, 2003). However, children with SLI need a greater number of exemplars before acquiring an abstract schema. For example, children with SLI need a greater number of types of verbs before abstracting morphological regularities and developing a more generalized knowledge about verbal categories (Conti-Ramsden \& Jones, 1997; Conti-Ramsden \& Windfuhr, 2002). Consequently, a greater number of exemplars could help children with SLI to detect relational structure and maybe still more with more complex items that require a heavier cognitive load. 


\section{Acknowledgment}

This work was supported by the Fonds de la Recherche Scientifique - FNRS (Belgium). We thank all the children who participated in this study, the speech and language therapists of the language class for their support and the teachers for their collaboration and their parents for their collaboration. We are grateful to Mélanie Guenebaud for her assistance in data collection. 


\section{References}

Benasich, A. A., \& Tallal, P. (2002). Infant discrimination of rapid auditory cues predicts later language impairment. Behavioural Brain Research, 136(1), 31-49.

Bishop, D. V., \& Norbury, C. F. (2005). Executive functions in children with communication impairments, in relation to autistic symptomatology 2: Response inhibition. Autism, 9(1), 2943.

Bybee, J. (2010). Language, usage and cognition. Cambridge, UK: Cambridge University Press.

Cho, S., Holyoak, K. J., \& Cannon, T. D. (2007). Analogical reasoning in working memory: Resources shared among relational integration, interference resolution, and maintenance. Memory \& Cognition, 35(6), 1445-1455.

Cohen, M. A., Evans, K. K., Horowitz, T. S., \& Wolfe, J. M. (2011). Auditory and visual memory in musicians and nonmusicians. Psychonomic Bulletin \& Review, 18(3), 586-591.

Cohen, M. A., Horowitz, T. S., \& Wolfe, J. M. (2009). Auditory recognition memory is inferior to visual recognition memory. PNAS Proceedings of the National Academy of Sciences of the United States of America, 106(14), 6008-6010.

Conti-Ramsden, G., \& Jones, M. (1997). Verb use in specific language impairment. Journal of Speech \& Hearing Research, 40(6), 1298-1313.

Conti-Ramsden, G., \& Windfuhr, K. (2002). Productivity with word order and morphology: A comparative look at children with $\mathrm{SLI}$ and children with normal language abilities. International Journal of Language \& Communication Disorders, 37(1), 17-30.

Costerman, J. (1980). Psychologie du Langage. Bruxelles: Mardaga.

Dunn, L. M., Theriault-Whalen, C. M., \& Dunn, L. M. (1993). Echelle de vocabulaire en images peabody. Toronto, ON: Psychan.

Elliott, L. L., Hammer, M. A., \& Scholl, M. E. (1989). Fine-grained auditory discrimination in normal children and children with language-learning problems. Journal of Speech \& Hearing Research, 32(1), 112-119.

Finneran, D. A., Francis, A. L., \& Leonard, L. B. (2009). Sustained attention in children with specific language impairment (SLI). Journal of Speech, Language, and Hearing Research, 52(4), 915929.

Friedman, N. P., \& Miyake, A. (2004). The Relations Among Inhibition and Interference Control Functions: A Latent-Variable Analysis. [Empirical Study; Quantitative Study]. Journal of Experimental Psychology: General, 133(1), 101-135.

Gabriel, A., Maillart, C., Stefaniak, N., Lejeune, C., Desmottes, L., \& Meulemans, T. (2013). Procedural learning in specific language impairment: Effects of sequence complexity. Journal of the International Neuropsychological Society, 19(3), 264-271.

Gentner, D. (1983). Structure-mapping: A theoretical framework for analogy. Cognitive Science, 7(2), 155-170.

Gentner, D., \& Colhoun, J. (2010). Analogical processes in human thinking and learning On Thinking: Vol. 2. Towards a Theory of Thinking.

Gentner, D., \& Markman, A. B. (1997). Structure mapping in analogy and similarity. American Psychologist, 52(1), 45-56.

Gentner, D., \& Namy, L. L. (2006). Analogical Processes in Language Learning. Current Directions in Psychological Science, 15(6), 297-301.

Gentner, D., \& Smith, L. (2012). Analogical reasoning. In V. Ramachandran (Ed.), Encyclopedia of Human Behavior (2nd ed.). Oxford, UK: Elsevier.

Goldberg, A. E. (1995). Constructions: A construction grammar approach to argument structure. Chicago: University of Chicago Press.

Goldberg, A. E. (2006). Constructions at work. The nature of generalization in language. Oxford: Oxford University Press. 
Goldwater, M. B., Tomlinson, M. T., Echols, C. H., \& Love, B. C. (2010). Structural priming as structureMapping: Children use analogies from previous utterances to guide sentence production. Cognitive Science: A Multidisciplinary Journal, 35(1), 156-170.

Gómez, R. L., \& Gerken, L. (2000). Infant artificial language learning and language acquisition. Trends in Cognitive Sciences, 4(5), 178-186.

Halford, G. S., \& Andrews, G. (2011). Information-processing models of cognitive development Goswami, Usha [Ed] (2011) The Wiley-Blackwell handbook of childhood cognitive development (2nd ed) ( $p$ p 697-721) xiii, 801 pp Wiley-Blackwell.

Im-Bolter, N., Johnson, J., \& Pascual-Leone, J. (2006). Processing Limitations in Children With Specific Language Impairment: The Role of Executive Function. Child Development, 77(6), 1822-1841.

Isaki, E., Spaulding, T. J., \& Plante, E. (2008). Contributions of language and memory demands to verbal memory performance in language-learning disabilities. Journal of Communication Disorders, 41(6), 512-530.

Johnston, J. R., \& Smith, L. B. (1989). Dimensional thinking in language impaired children. [Empirical Study]. Journal of Speech \& Hearing Research, 32(1), 33-38.

Jones, M., \& Conti-Ramsden, G. (1997). A comparison of verb use in children with Specific Language Impairment and their younger siblings. Journal of Speech Language and Hearing Research, 40, 1298-1313.

Kamhi, A. G., Gentry, B., Mauer, D., \& Gholson, B. (1990). Analogical learning and transfer in language-impaired children. Journal of Speech \& Hearing Disorders, 55(1), 140-148.

Khomsi, A. (2001). ELO: évaluation du langage oral. Paris, France: ECPS.

Kroll, J. F., \& Potter, M. C. (1984). Recognizing words, pictures, and concepts: A comparison of lexical, object, and reality decisions. Journal of Verbal Learning and Verbal Behavior, 23, 39-66.

Langacker, R. W. (1987). Foundation of Cognitive Grammar (Vol. 1). Standford, CA: Standford University Press.

Lecocq, P. (1996). Epreuve de Compréhension Syntaxico-Sémantique. Villeneuve-d'Ascq, France: Presses universitaires du Septentrion.

Leonard, L. B. (1998). Children with specific language impairment. Cambridge, MA: The MIT Press.

Leonard, L. B., Weismer, S. E., Miller, C. A., Francis, D. J., Tomblin, J., \& Kail, R. V. (2007). Speed of processing, working memory, and language impairment in children. Journal of Speech, Language, and Hearing Research, 50(2), 408-428.

Leroy, S., Parisse, C., \& Maillart, C. (2012). Analogical reasoning in children with specific language impairment. Clinical Linguistics \& Phonetics, 26(4), 380-395.

Loewenstein, J., \& Gentner, D. (2001). Spatial mapping in preschoolers: Close comparisons facilitate far mappings. Journal of Cognition and Development, 2(2), 189-219.

Marcus, G., Vijayan, S., Rao, S., \& Vishton, P. (1999). Rule learning by seven-month-old infants. Science, 283(5398), 77-80.

Markman, A. B., \& Gentner, D. (1993). Structural alignment during similarity comparisons. Cognitive Psychology, 25(4), 431-467.

Marshall, J. (2003). Noun-verb dissociations--Evidence from acquisition and developmental acquired impairments. Journal of Neurolinguistics, 16(2-3), 67-84.

Marton, K., Kelmenson, L., \& Pinkhasova, M. (2007). Inhibition control and working memory capacity in children with SLI. Psychologia: An International Journal of Psychology in the Orient, 50(2), 110-121.

Masterson, J. J., Evans, L. H., \& Aloia, M. (1993). Verbal analogical reasoning in children with language-learning disabilities. Journal of Speech \& Hearing Research, 36(1), 76-82.

Masterson, J. J., \& Perrey, C. D. (1999). Training analogical reasoning skills in children with language disorders. American Journal of Speech-Language Pathology, 8, 53-61.

Montgomery, J. W., \& Evans, J. L. (2009). Complex sentence comprehension and working memory in children with specific language impairment. Journal of Speech, Language, and Hearing Research, 52(2), 269-288. 
Morrison, R. G., Doumas, L. A., \& Richland, L. E. (2006). The development of analogical reasoning in children: A computational account. Paper presented at the Proceedings of the Twenty-Eighth Annual Conference of the Cognitive Science Society, Mahwah, NJ.

Morrison, R. G., Holyoak, K. J., \& Truong, B. (2001). Working memory modularity in analogical reasoning. Paper presented at the Twenty-fourth Annual Conference of the Cognitive Science Society Mahwah, NJ.

Nippold, M. A., Erskine, B. J., \& Freed, D. B. (1988). Proportional and functional analogical reasoning in normal and language-impaired children. Journal of Speech \& Hearing Disorders, 53(4), 440448.

Oram Cardy, J. E., Tannock, R., Johnson, A. M., \& Johnson, C. J. (2010). The contribution of processing impairments to SLI: Insights from attention-deficit/hyperactivity disorder. Journal of Communication Disorders, 43(2), 77-91.

Rattermann, M. J., \& Gentner, D. (1998). More evidence for a relational shift in the development of analogy: children's performance on a causal-mapping task. Cognitive Development, 13, 453478.

Riches, N., Faragher, B., \& Conti-Ramsden, G. (2006). Verb schema use and input dependence in 5year-old children with specific language impairment (SLI). International Journal of Language \& Communication Disorders, 41(2), 117-135.

Richland, L. E., Chan, T.-K., Morrison, R. G., \& Au, T. K. (2010). Young children's analogical reasoning across cultures: Similarities and differences. Journal of Experimental Child Psychology, 105(12), 146-153.

Richland, L. E., Morrison, R. G., \& Holyoak, K. J. (2006). Children's development of analogical reasoning: Insights from scene analogy problems. Journal of Experimental Child Psychology, 94(3), 249-273.

Saffran, J. R., Pollak, S. D., Seibel, R. L., \& Shkolnik, A. (2007). Dog is a dog is a dog: Infant rule learning is not specific to language. Cognition, 105(3), 669-680.

Schneider, W., Eschman, E., \& Zuccolotto, A. (2002). E-Prime user's guide. Pittsburgh: Psychology Software Tools Inc.

Schwartz, R. G. (2009). Specific language impairment. In R. G. Schwartz (Ed.), Handbook of child language disorders (pp. 3-43). New York, NY: Psychology Press.

Skipp, A., Windfuhr, K. L., \& Conti-Ramsden, G. (2002). Children's grammatical categories of verb and noun: A comparative look at children with specific language impairment (SLI) and normal language (NL). International Journal of Language \& Communication Disorders, 37(3), 253271.

Stark, R. E., \& Hienz, J. (1996). Vowels perception in language impaired and language normal children. Journal of Speech \& Hearing Research, 39, 860-869.

Sussman, J. (1993). Perception of formant transition cues to place of articulation in children with language impairments. Journal of Speech and Hearing Research, 36, 1289-1309.

Tallal, P. (1990). Fine-grained discrimination deficits in language-learning impaired children are specific neither to the auditory modality nor to speech perception. [Comment/Reply]. Journal of Speech \& Hearing Research, 33(3), 616-617.

Thibaut, J.-P., French, R., \& Vezneva, M. (2010). The development of analogy making in children: Cognitive load and executive functions. Journal of Experimental Child Psychology, 106(1), 119.

Thordardottir, E., \& Weismer, S. E. (2002). Verb argument structure weakness in specific language impairment in relation to age and utterance length. Clinical Linguistics \& Phonetics, 16(4), 233-250.

Tomasello, M. (2000). Do young children have adult syntactic competence? Cognition, 74(3), 209253.

Tomasello, M. (2003). Constructing a language: A usage-based theory of language acquisition. Cambridge, MA: Harvard University Press. 
Viskontas, I., Morrison, R. G., Holyoak, K. J., Hummel, J. E., \& Knowlton, B. J. (2004). Relational Integration, Inhibition, and Analogical Reasoning in Older Adults. Psychology and Aging, 19(4), 581-591.

Vugs, B., Cuperus, J., Hendriks, M., \& Verhoeven, L. (2013). Visuospatial working memory in specific language impairment: a meta-analysis. Research in Developmental Disabilities, 34, 25862597.

Wechsler, D. (2005). Wechsler Intelligence Scale for Children - Fourth Edition. San Antonio, TX: Harcourt Assessment.

Whitehouse, A. J. (2010). Is there a sex ratio difference in the familial aggregation of specific language impairment? A meta-analysis. [Literature Review; Systematic Review]. Journal of Speech, Language, and Hearing Research, 53(4), 1015-1025. 